\title{
Growth medium composition-determined regulatory mechanisms are superimposed on CatR-mediated transcription from the pheBA and catBCA promoters in Pseudomonas putida
}

\author{
Andres Tover, Eve-Ly Ojangu and Maia Kivisaar
}

\begin{abstract}
Author for correspondence: Maia Kivisaar. Tel: +372 7 375015. Fax: + 3727420286. e-mail:maiak@ebc.ee
\end{abstract}

Department of Genetics, Institute of Molecular and Cell Biology, Estonian Biocentre and Tartu University, Riia 23, 51010 Tartu, Estonia
Expression of the phenol degradation pathway in Pseudomonas putida strain PaW85 requires coordinated transcription of the plasmid-borne pheBA operon encoding catechol 1,2-dioxygenase and phenol monooxygenase, respectively, and the chromosomally encoded catechol degradation catBCA operon. Transcriptional activation from the pheBA and catBCA promoters is regulated by CatR and the catechol degradation pathway intermediate cis,cis-muconate. Here it is shown that physiological control mechanisms are superimposed on this regulatory system. Transcriptional activation from the pheBA and catBCA promoters is growth-phase-regulated in $P$. putida cells grown on rich medium (LB medium). CatR-mediated transcription from these promoters is silenced on rich medium until the transition from exponential to stationary phase. A slight positive effect (threefold) of stationary-phase-specific sigma factor $\sigma^{\mathrm{s}}$ on transcription from the pheBA promoter was observed. Expression of the catBCA promoter was not influenced by the activity of this sigma factor. In contrast to rich growth medium, transcription from the pheBA and catBCA promoters in minimal medium containing a mixture of glucose and sodium benzoate was rapidly induced in exponential culture. It was shown that the presence of amino acids in the culture medium causes exponential silencing of the pheBA and catBCA promoters. The possibility that a hypothetical repressor protein could be involved in physiological control of transcription from the pheBA and catBCA promoters is discussed.

Keywords: pheBA and catBCA operons, exponential silencing of transcription

\section{INTRODUCTION}

Expression of catabolic pathways in Pseudomonas is inhibited by a number of growth conditions that adjust the activity of specific promoters to a given metabolic and physiological status (Cases \& de Lorenzo, 1998). The best studied examples of growth-condition-dependent expression of aromatic compound degradation genes in soil pseudomonads are catabolic operons of TOL plasmid pWW0 (Cases \& de Lorenzo, 2000; Marques et al., 1994, 1999; Ramos et al., 1997 and references cited therein) and dimethylphenol degradation genes, $d m p$, from megaplasmid pVI150 (Sze \& Shingler, 1999). The strong repressive effect of LB

Abbreviations: $\mathrm{CCM}, \mathrm{cis}$, cis-muconate; $\beta$-Gal, $\beta$-galactosidase. medium or amino acids has been observed for the promoters of these operons (Cases et al., 1996; de Lorenzo et al., 1993; Sze \& Shingler, 1999; Sze et al., 1996). The physiological down-regulation of the $\mathrm{Pu}$ promoter of TOL plasmids is a complex process controlled by two or more independent mechanisms (Cases \& de Lorenzo, 2000). For example, PtsNmediated carbon source inhibition (Cases et al., 1999) and the control of the activity of $\sigma^{54}$ (Cases et al., 1996) are involved in regulation of transcription from $\mathrm{Pu}$. Promoter Po of the $d m p$ operon drives the expression of the dimethylphenol degradation pathway very similarly to that of $\mathrm{Pu}$ for the upper pathway of TOL plasmids (Sze et al., 1996). The stimulating effect of (p)ppGpp on transcriptional activation was recently demonstrated for Po (Carmona et al., 2000). At the same time, this signal molecule, which triggers the stringent response to 


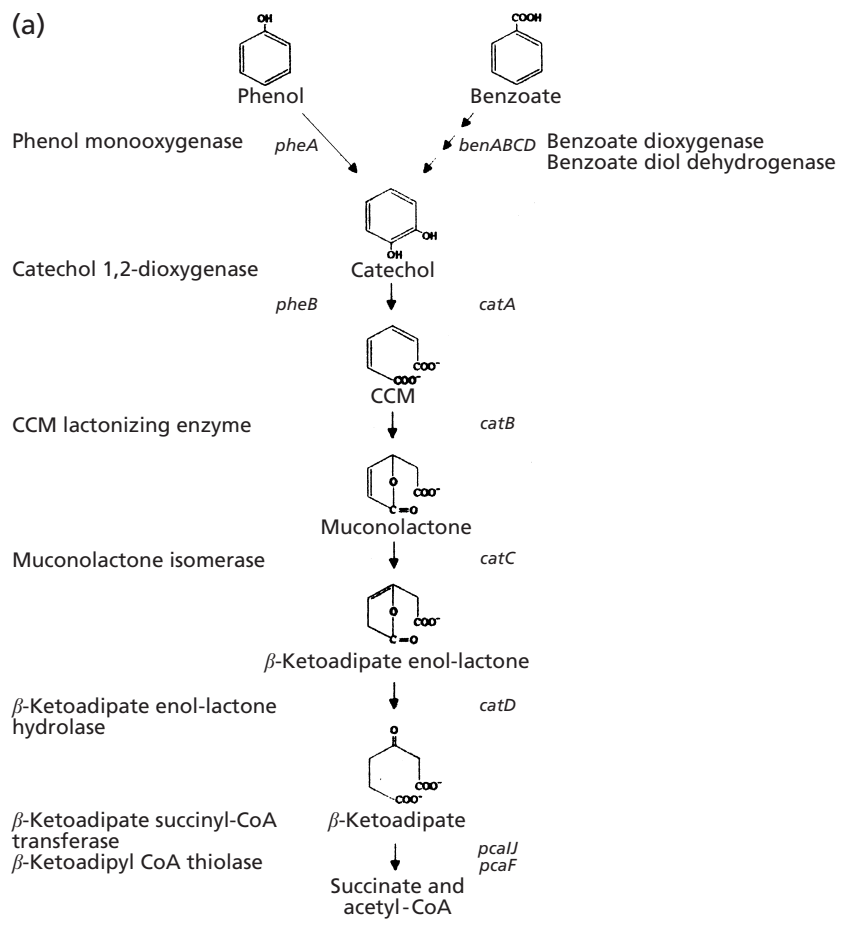

(b)

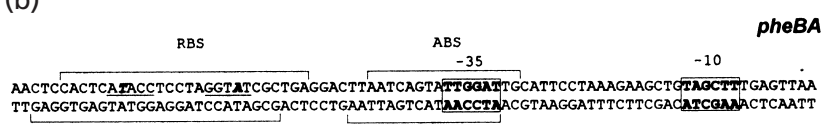
TTGAGGTGAGTATGGAGGATCCATAGCGACTCCTGAATTAGTCATAMCCTNACGTAAGGATTTCTTCGACATCONNACTCAATT

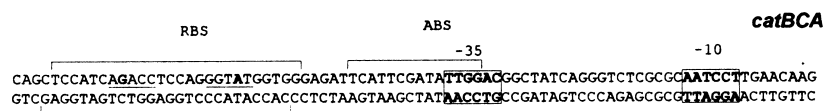
GTCGAGGTAGTCTGGAGGTCCCATACCACCCTCTAAGTAAGCTATAACCTECCGATAGTCCCAGAGCGCGIIAGGAACTTGTTC

Fig. 1. (a) The $\beta$-ketoadipate pathway by which phenol and benzoate are converted to catechol and to the common metabolic intermediates succinate and acetyl-CoA. (b) Schematic representation of the promoter region of the pheBA and cat $B C A$ operons. The bracketed sequences represent nucleotides protected by CatR from cleavage by DNasel (Chugani et al., 1997; Parsek et al., 1995). The protected regions are designated as RBS (recognition binding sites) and ABS (activation binding sites). Bold/italic type designates the $T$ and $A$ residues of the conserved $T-\mathrm{N}_{11}-A$ sequence of the Lys $R$ family regulatory proteins binding motif of the RBS. The catBCA promoter has a $\mathrm{G}$ instead of the conserved $\mathrm{T}$. The nucleotides of the interrupted inverted repeat within the RBS are underlined. The nucleotides of the -35 and -10 hexamers of the promoters are in bold type and boxed. The transcriptional start sites are denoted by asterisks.

amino acid starvation, had only a moderate effect on the activity of the very similar promoter $\mathrm{Pu}$ (Carmona et al., 2000).

In Pseudomonas species, phenolic compounds are transformed by different enzymes to central intermediates, such as protocatechuate and (substituted) catechols (Harayama \& Timmis, 1989). Typically, unsubstituted compounds, such as benzoate, are metabolized by an ortho-cleavage pathway (Fig. 1). The genes for benzoate metabolism, including ortho-pathway genes, are chromosomally encoded in P. putida (Harwood \& Parales, 1996). The catBCA operon encodes three enzymes of the ortho-pathway required for benzoate catabolism, namely muconate lactonizing enzyme I, muconolactone isomerase and catechol 1,2-dioxygenase, respectively (Houghton et al., 1995). The induction of this operon, which is $\sigma^{54}$-independent, requires a LysR family transcriptional activator, CatR, and an inducer molecule, cis,cis-muconate (CCM), an intermediate of the ortho-pathway (Rothmel et al., 1990, 1991). The pheB and pheA genes originating from plasmid DNA of Pseudomonas sp. EST1001 encode catechol 1,2-dioxygenase and phenol monooxygenase, respectively (Kivisaar et al., 1990). When the pheBA operon is introduced into $P$. putida, the bacteria acquire the ability to degrade phenol (Kivisaar et al., 1991, 1990). The pheBA promoter resembles the catBCA promoter and is also activated by CatR (Kasak et al., 1993; Parsek et al., 1995). Comparative studies of the interaction of CatR with the promoters of the pheBA and catBCA operons have revealed that the CatRmediated activation mechanism is well conserved, despite the different origins of these operons (Parsek et al., 1995; Tover et al., 2000).

In this study we have investigated (i) how the physiological status of the cells controls expression of the pheBA and catBCA promoters in P. putida and (ii) the similarity of these control mechanisms on transcription from these promoters. We show that transcription from the pheBA and catBCA promoters is strongly influenced by the composition of the growth medium (rich versus minimal medium). The presence of amino acids in the growth medium is one component that causes exponential silencing of both the pheBA and the catBCA promoters.

\section{METHODS}

Bacterial strains and plasmids. The bacterial strains and plasmids used in this study are listed in Table 1. Escherichia coli TG1 (Carter et al., 1985) was used for DNA cloning procedures. E. coli was incubated at $37^{\circ} \mathrm{C}$ and P. putida at $30^{\circ} \mathrm{C}$. E. coli was transformed with plasmid DNA as described by Hanahan (1983). P. putida was electrotransformed according to the protocol described by Sharma \& Schimke (1996). Antibiotics were added at the following final concentrations: ampicillin at $100 \mu \mathrm{g} \mathrm{ml}^{-1}$ for E. coli; carbenicillin at $1500 \mu \mathrm{g} \mathrm{ml}^{-1}$ and kanamycin at $50 \mu \mathrm{g} \mathrm{ml}^{-1}$ for P. putida.

Growth conditions and $\beta$-galactosidase ( $\beta$-Gal) assay. To investigate the role of rich medium on transcription from the pheBA and catBCA promoters, $P$. putida cells were grown in LB medium (Miller, 1992). Cells were grown overnight in LB medium and diluted into fresh medium to obtain an $\mathrm{OD}_{580}$ of $0 \cdot 02$. After $2 \mathrm{~h}$ cultivation sodium benzoate was added at a final concentration of $2.5 \mathrm{mM}$ to induce the transcription of the pheBA-lacZ and catBCA-lacZ transcriptional fusions. The inducer molecule, CCM, required for CatR-dependent transcriptional activation is an intermediate of the benzoate degradation pathway. Because $P$. putida uses sodium benzoate as a carbon source, this compound was added again after 5 
Table 1. Bacterial strains and plasmids used in this study

\begin{tabular}{|c|c|c|}
\hline Strain or plasmid & Genotype or construction & Source of reference \\
\hline \multicolumn{3}{|l|}{ E. coli } \\
\hline TG1 & supE hsd $\Delta 5$ thi $\Delta\left(\right.$ lac-proAB) $\mathrm{F}^{\prime}\left(\right.$ traD36 proAB lacl $^{\mathrm{a}}$ lacZ $\left.\Delta \mathrm{M} 15\right)$ & Carter et al. (1985) \\
\hline S17-1 $\lambda$ pir & $\mathrm{Tp}^{\mathrm{r}} \mathrm{Sm}^{\mathrm{r}}$ recA thi pro $\left(\mathrm{r}^{-} \mathrm{m}^{+}\right) \mathrm{RP} 4:: 2 \mathrm{Tc}:: \mathrm{Mu}:: \mathrm{Km} \mathrm{Tn} 7 \lambda$ pir & Miller \& Mekalanos (1988) \\
\hline C118 $\lambda$ pir & $\begin{array}{l}\Delta(\text { ara-leu }) \text { araD } \Delta \text { lacX } 74 \text { galE galK phoA20 thi-1 rpsE rpoB argE (Am) } \\
\text { recA1 } \lambda \text { pir phage lysogen }\end{array}$ & Herrero et al. (1990) \\
\hline \multicolumn{3}{|c|}{ 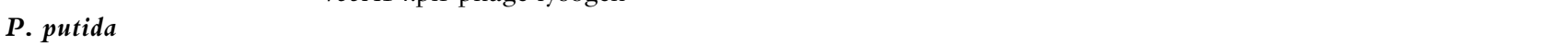 } \\
\hline $\mathrm{PaW} 85$ & $\operatorname{Tn} 4652$ & Bayley et al. (1977) \\
\hline PKS54 & $\operatorname{Tn} 4652$ rpos: : $\mathrm{Km}^{\mathrm{r}}$ & Ojangu et al. (2000) \\
\hline $\mathrm{PaW} 85 \mathrm{CatR}^{+}$ & $\begin{array}{l}\text { PaW85 catR gene under control of Ptac promoter and } l a c I^{\alpha} \text { repressor, } \\
\mathrm{Km}^{\mathrm{r}}\end{array}$ & This study \\
\hline \multicolumn{3}{|c|}{ The } \\
\hline pBluescript SK $(+)$ & Cloning vector $\left(\mathrm{Ap}^{\mathrm{r}}\right)$ & Stratagene \\
\hline pKTlacZ & Cloning vector $\left(\mathrm{Ap}^{\mathrm{r}}\right)$ & Hõrak \& Kivisaar (1998) \\
\hline pZ-pheBA & pheBA promoter cloned into pKTlacZ & This study \\
\hline $\mathrm{pZ}$-catBCA & catBCA promoter cloned into $\mathrm{pKTlacZ}$ & This study \\
\hline pUTmini-Tn5 Km2 & Delivery plasmid for mini $\operatorname{Tn} 5 \mathrm{Km} 2$ & de Lorenzo et al. (1993) \\
\hline pUC18Not & pUC18 with NotI restriction sites in multicloning region $\left(\mathrm{Ap}^{\mathrm{r}}\right)$ & Herrero et al. (1990) \\
\hline pBRlacItac & $\begin{array}{l}\text { Ptac promoter and } l a c I^{\mathrm{a}} \text { repressor in } 2 \cdot 2 \mathrm{~kb} N r u \mathrm{I}-E c o \mathrm{RI} \text { fragment from } \\
\text { plasmid pMMB208 cloned into EcoRV/EcoRI-cleaved pBR322 }\end{array}$ & Ojangu et al. (2000) \\
\hline $\mathrm{pKZ} \Delta \mathrm{Hf}$ & pKR1300 derivative containing cat $R$ gene & Rothmel et al. (1990) \\
\hline pBRlacItac-catR & $\begin{array}{l}\text { catR in } 1 \mathrm{~kb} E c o \mathrm{RI}-H i n d I I I \text { fragment cloned into pBRlacItac to obtain } \\
\text { lacl }{ }^{\mathrm{q}}-\mathrm{P} t a c-c a t R\end{array}$ & This study \\
\hline pUCPtac-catR & lacl ${ }^{\mathrm{a}}$-Ptac-catR expression cassette cloned into pUC18Not & This study \\
\hline pUT-lacItac-catR & $\begin{array}{l}\text { lacl }{ }^{\mathrm{a}}-\mathrm{P} t a c-c a t R \text { expression cassette cloned from pUCPtac-catR into } \\
\text { pUT-miniTn } 5 \mathrm{Km} 2\end{array}$ & This study \\
\hline pVI536 & relA gene of E. coli overexpressed from $\mathrm{P}_{\mathrm{BAD}}$ & Sze \& Schingler (1999) \\
\hline
\end{tabular}

and $12 \mathrm{~h}$ cultivation (at a final concentration of $5 \mathrm{mM}$ ) to maintain the source of the inducer in the growth medium. Samples for the $\beta$-Gal assay were taken from cultures grown for 5,12 and $24 \mathrm{~h}$.

To investigate the regulation of transcription from the $p h e B A$ and catBCA promoters in minimal medium, $P$. putida cells were grown overnight in MOPS medium (Neidhardt et al., 1974) either with $0.5 \%$ CAA or amino acids at a final concentration of $200 \mathrm{ng}$ each amino acid $\mathrm{ml}^{-1}$. The overnight bacterial culture was diluted with fresh medium $(1: 20, \mathrm{v} / \mathrm{v})$ grown to exponential phase and then diluted again in minimal medium containing $0 \cdot 2 \%$ glucose. The $\mathrm{OD}_{580}$ of the diluted culture was $0 \cdot 1$. Sodium benzoate was added at a final concentration of $10 \mathrm{mM}$ at the beginning of the experiment and $1 \mathrm{ml}$ samples were taken at different time points during growth to assay the $\beta$-Gal activity in cell suspensions.

To investigate the possible effect of overexpression of the CatR protein on the transcription from the pheBA and cat $B C A$ promoters, the culture was grown overnight in LB medium and thereafter diluted into fresh LB medium as indicated above. To induce the expression of CatR, IPTG (at a final concentration of $0.5 \mathrm{mM}$ ) was added to the growth medium. When the culture reached the exponential growth phase, it was diluted again into fresh LB medium containing $0.5 \mathrm{mM}$ IPTG. At this step, $5 \mathrm{mM}$ sodium benzoate was added for the induction of transcription from the pheBA and catBCA promoters.
A $\beta$-Gal assay with the cell lysates was carried out as specified by Miller (1992). In all cases at least three independent measurements were made. Protein concentration in crude lysates was measured by the method of Bradford (1976). $\beta$-Gal measurement in cell suspensions was performed by modification of the standard protocol of Miller (1992). The amount of $\mathrm{Z}$ buffer $\left(60 \mathrm{mM} \mathrm{Na} \mathrm{HPO}_{4}, 40 \mathrm{mM} \mathrm{NaH} \mathrm{PO}_{4}\right.$, $10 \mathrm{mM} \mathrm{KCl}, 1 \mathrm{mM} \mathrm{MgSO}_{4}$ and $50 \mathrm{mM} \beta$-mercaptoethanol) in a test tube was $1.6 \mathrm{ml}$. ONGP (2-nitrophenyl- $\beta$-D-galactopyranoside; $400 \mu \mathrm{l}$ of a $50 \mathrm{mg} \mathrm{ml}^{-1}$ solution), $100 \mu \mathrm{l} 0.002 \%$ SDS and $100 \mu \mathrm{l}$ chloroform were added into the reaction mixture. Finally, bacteria were added into the test tube and mixed well. The $\beta$-Gal reaction was stopped by the addition of $1 \mathrm{ml} 1 \mathrm{M} \mathrm{Na} \mathrm{NaO}_{3}$. Data of at least three independent experiments are presented in all figures.

Cloning procedures. Construction of the pheBA promoter-lacZ transcriptional fusion (plasmid pZ-pheBA) was described previously (Tover et al., 2000). For the cloning of the catBCA promoter into the promoter-probe plasmid pKTlacZ (Hõrak \& Kivisaar, 1998), the 207 bp catBCA promoter region from the $P$. putida chromosome was amplified by using oligonucleotides cat35 (5'-GGGCTGCCAGCCGCGGGCCC-3') and AFC62 [5'-AGCGCGGCGGCTCGACGACGCTG(PstI)CAGAGC-3'], complementary to the upstream and downstream regions of the catBCA promoter, respectively. The PCR amplification product was cleaved with PstI and cloned into pBluescript SK $(+)$ cleaved with Pst I and EcoRV. The sequence of the catBCA promoter was verified by DNA sequencing. Subsequently, the catBCA 
promoter was inserted using BamHI- and XhoI-generated ends into the promoter-probe vector $\mathrm{pKTlacZ}$ to obtain plasmid pZ-catBCA. For the construction of $P$. putida CatR overexpression strain $\mathrm{PaWCatR}^{+}$, the catR gene was cloned from plasmid pKR $\Delta \mathrm{HF}$ (Rothmel et al., 1991) by using HindIII- and EcoRI-generated ends to the vector pBRlacItac (Ojangu et al., 2000) cleaved with same enzymes (pBRlacItaccatR in Table 1). The CatR expression cassette lacl $^{\mathrm{q}}$-Ptac-catR was inserted into pUC18Not (Herrero et al., 1990) using EcoRI- and BamHI-generated ends. Thereafter, the lacI ${ }^{\mathrm{a}}-\mathrm{Ptac}$ cat $R$ sequence was inserted into the Not I site of pUTmini-Tn5 $\mathrm{Km} 2$ (de Lorenzo et al., 1990) and pUTlacItac-catR was selected in E. coli C118 גpir (Herrero et al., 1990). The lacl ${ }^{\mathrm{a}}$-Ptac-catR expression cassette was inserted into the chromosome of $P$. putida strain PaW85 (Bayley et al., 1977) by the method of random insertion using E. coli S17-1 $\lambda$ pir (Miller \& Mekalanos, 1988) as donor strain. P. putida PaW85CatR ${ }^{+}$ was selected at $30^{\circ} \mathrm{C}$ on glucose/kanamycin plates. The expression of CatR in $\mathrm{PaW} 85 \mathrm{CatR}^{+}$was verified by Western blot analysis using polyclonal antibodies against $P$. putida CatR protein.

\section{RESULTS AND DISCUSSION}

\section{Growth-phase-dependent activation of transcription of the pheBA and catBCA operons}

The promoters of the $p h e B A$ and $c a t B C A$ operons were cloned into plasmid pKTlacZ carrying the lac $Z$ reporter gene and $\beta$-Gal activity was measured to examine transcription from these promoters. P. putida PaW85 cells containing lac $Z$ transcriptional fusion plasmids pZ-pheBA and pZ-catBCA were grown in rich medium (LB) to the late stationary phase. $\beta$-Gal activity was measured in cells sampled from different time points during culture (Fig. 2). Sodium benzoate was always added into the growth medium to induce transcription from the pheBA and cat $B C A$ promoters. Results presented in Fig. 2 show that transcription from the pheBA and catBCA promoters remained at a low level during the first $9 \mathrm{~h}$ of growth. The level of expression of $\beta$-Gal activity was remarkably elevated in stationary-phase cells and it remained high in stationary-phase cultures during the $24 \mathrm{~h}$ period of the study. This indicated that the pheBA and catBCA promoters are stationary-phaseinducible and down-regulated during exponential growth.

\section{Transcription from the phe $B A$ and cat $B C A$ promoters in a $\sigma^{\mathrm{s}}$-deficient background}

Use of an in vitro transcription assay has demonstrated that the only requirements for transcription from the pheBA and catBCA promoters are purified CatR, CCM and RNA polymerase- $\sigma^{70}$ holoenzyme $\left(\mathrm{E} \sigma^{70}\right)$ (Chugani et al., 1997). Stationary-phase-specific sigma factor $\sigma^{\mathrm{S}}$, encoded by $r p o S$, activates transcription of several genes in stationary phase or otherwise stressed bacteria (Hengge-Aronis, 1999). Analysis of $\sigma^{70}$ - and $\sigma^{\mathrm{S}}$-promoter specificity has revealed that $\mathrm{E} \sigma^{70}$ and $\mathrm{E} \sigma^{\mathrm{S}}$ recognize many promoter sequences equally well in vitro, but differentially control genes in vivo (reviewed by Hengge-Aronis, 2000). The exponential silencing of transcription from (a)

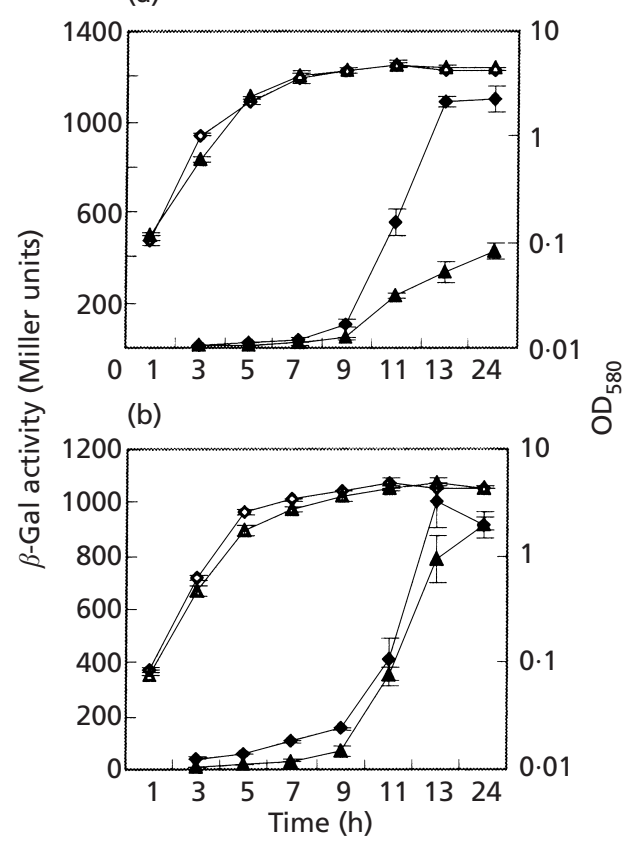

Fig. 2. Effect of the growth phase of bacteria and $\sigma^{s}$ on transcription from the pheBA promoter (a) and from the catBCA promoter (b) in P. putida strain PaW85 (wild-type; $\bullet$, $\diamond)$ and in its rpoS-defective derivative PKS54 $(\boldsymbol{\Lambda}, \triangle)$. $\beta$-Gal activity (filled symbols) was measured either in cells of $P$. putida strain PaW85 (wild-type) or the rpoS-deficient strain PKS54 harbouring either of the plasmids pZ-pheBA or pZ-catBCA. Bacteria were grown in LB medium to stationary phase in the presence of sodium benzoate. The growth curves of $P$. putida cells are indicated by open symbols. The standard deviation is shown in the figure.

the pheBA and catBCA promoters gave us the idea to investigate the role of $\sigma^{\mathrm{s}}$ on transcription from these promoters.

We have previously constructed a $P$. putida $\sigma^{\mathrm{s}}$-deficient strain, PKS54, that is a derivative of the wild-type strain PaW85 (Ojangu et al., 2000). Plasmids pZ-pheBA and pZ-catBCA, containing the pheBA-lacZ and catBCAlac $Z$ transcriptional fusions, respectively, were introduced into the rpoS-deficient strain, PKS54, and into the wild-type strain, PaW85. The level of expression of $\beta$ Gal activity measured in exponential- and stationaryphase cells of PKS54 was compared with that estimated in PaW85. The results presented in Fig. 2 show that in the case of the pheBA promoter, $\beta$-Gal activity remained approximately threefold lower in the $\sigma^{\mathrm{s}}$ deficient strain than in the wild-type strain in stationaryphase cultures (Fig. 2a). At the same time, lack of expression of $\sigma^{\mathrm{S}}$ in $P$. putida cells did not affect transcription from the catBCA promoter (Fig. 2b). The sequence of the -10 region of the cat $B C A$ promoter (CAATCCT) shows more similarity to the consensus CTATACT proposed for the promoters recognized by $\mathrm{E} \sigma^{\mathrm{S}}$ than that of the pheBA promoter (CTAGCTT). Based on in vivo experiments presented in this paper we 


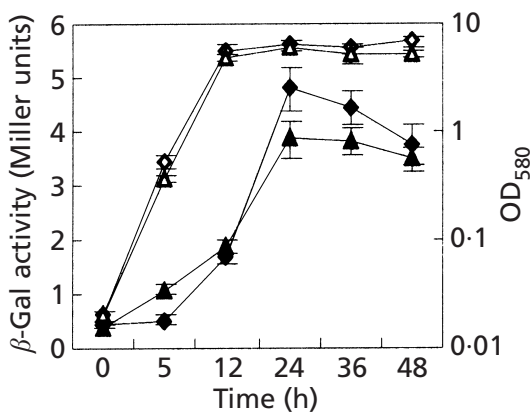

Fig. 3. Effect of the growth phase of bacteria and $\sigma^{\mathrm{s}}$ on transcription from the catR promoter. $\beta$-Gal activity (filled symbols) was measured either in the cell lysates of $P$. putida strain PaW85 (wild-type; $\diamond, \diamond)$ or in rpoS-deficient strain PKS54 $(\boldsymbol{\Delta}, \triangle$ ) harbouring the catR-lacZ fusion in plasmid pKTlacZ. Bacteria were grown in LB medium to stationary phase in the presence of sodium benzoate. The growth curves of $P$. putida cells are indicated by open symbols. The standard deviation is shown in the figure.

cannot state that the pheBA promoter is recognized by $\sigma^{\mathrm{S}}$. However, the nucleotide sequence of the -10 region of the promoter is only one component that determines $\sigma^{\mathrm{s}}$-dependent transcription. There is increasing evidence that additional regulators play a crucial role in establishing sigma factor specificity at stress-inducible promoters (Hengge-Aronis, 1999). Moreover, coming back to the sequence determinants, we have recently shown on fusion promoters generated in a starving population of $P$. putida that not only the -10 hexameric sequence, but also sequence downstream from the -10 hexamer is important for $\sigma^{\mathrm{s}}$-dependent transcription (Ojangu et al., 2000).

The positive effect of $\sigma^{\mathrm{S}}$ observed in the case of the pheBA promoter can give $P$. putida cells a little advantage to use phenol as a single source of carbon and energy under stressful conditions. However, this effect is insufficient to account for the inhibition of the pheBA promoter during exponential growth. Moreover, transcription from the catBCA promoter was not influenced by the presence of $\sigma^{\mathrm{S}}$ in $P$. putida cells. It is obvious, therefore, that stationary-phase-specific transcription from the pheBA and catBCA promoters must be regulated by some other mechanism than $\sigma^{\mathrm{S}}$-mediated control.

\section{Modulation of pheBA and catBCA promoter activity by growth phase does not operate through the amount of regulator protein CatR}

Transcriptional activation from the pheBA and catBCA promoters requires the presence of CatR and an inducer molecule CCM (Kasak et al., 1993; Parsek et al., 1995). Therefore, we examined whether the amount of the regulator protein CatR would be limiting in transcriptional activation from the pheBA and catBCA promoters in exponentially growing cells. To study the expression of the cat $R$ gene during the growth cycle, the promoter of the cat $R$ gene was cloned into plasmid pKTlacZ to generate a catR-lacZ transcriptional fusion. The activity of this fusion was compared in the wildtype P. putida strain PaW85 and in its rpoS-deficient mutant PKS54. In both strains, the level of $\beta$-Gal expression increased in stationary-phase cells when compared to that observed in exponentially growing cells (Fig. 3). Therefore, although cat $R$ promoter activity remained at a very low level during all growth phases of the bacteria, the intracellular amount of CatR (undetectable by Western blot analysis) may be somewhat increased in stationary-phase cells.

To study whether exponential silencing of the pheBA and cat $B C A$ promoters could be related to the amount of CatR in cells, we constructed a $P$. putida strain enabling artificial modulation of the expression of the $c a t R$ gene. For that purpose, the cat $R$ gene was cloned under the control of inducible Ptac promoter and the $\operatorname{lacl}^{\mathrm{q}}$ repressor. The cat $\mathrm{R}$ expression cassette was introduced into the chromosome of $P$. putida PaW85. Overexpression of CatR in the presence of $0.5 \mathrm{mM}$ IPTG was verified by using Western blot analysis with polyclonal antibodies against CatR (data not shown). We found that overexpression of CatR does not affect transcription from the $p h e B A$ and catBCA promoters in stationary-phase cells. No transcription from these promoters could be detected in exponentially growing cells (sampled from 2-h-old cultures), irrespective of the addition of IPTG into the growth medium. Therefore we concluded that the exponential silencing of the $p h e B A$ and $c a t B C A$ promoters is not determined by the cellular amount of CatR.

\section{Exponential silencing of the pheBA and catBCA promoters is dependent on the nature of the culture medium}

The role of growth medium in the regulation of gene expression has been shown in many cases. For example, growth medium composition (either rich or minimal) determines the level of transcription from the Po promoter of the operon $(d m p)$ encoding dimethylphenol degradation (Sze \& Shingler, 1999; Sze et al., 1996), from the $\mathrm{Pu}$ promoter of the TOL plasmid pWW0 (Cases et al., 1996; de Lorenzo et al., 1993) and from the PalkB promoter from the Pseudomonas oleovorans alkane degradation pathway (Yuste et al., 1998). We found that transcription from the pheBA and catBCA promoters was rapidly activated when bacteria were grown in MOPS minimal medium (Fig. 4). Measurement of $\beta$-Gal activity in cells sampled at different time points of an exponentially growing culture of $P$. putida PaW85 allowed us to detect the enzyme activity as early as $20 \mathrm{~min}$ after the addition of sodium benzoate.

When $0.5 \%$ CAA was added to minimal medium, the activation of transcription from the pheBA and catBCA promoters was delayed, similar to that observed in cells grown in LB medium (compare Figs 2 and 4). In the 
(a)

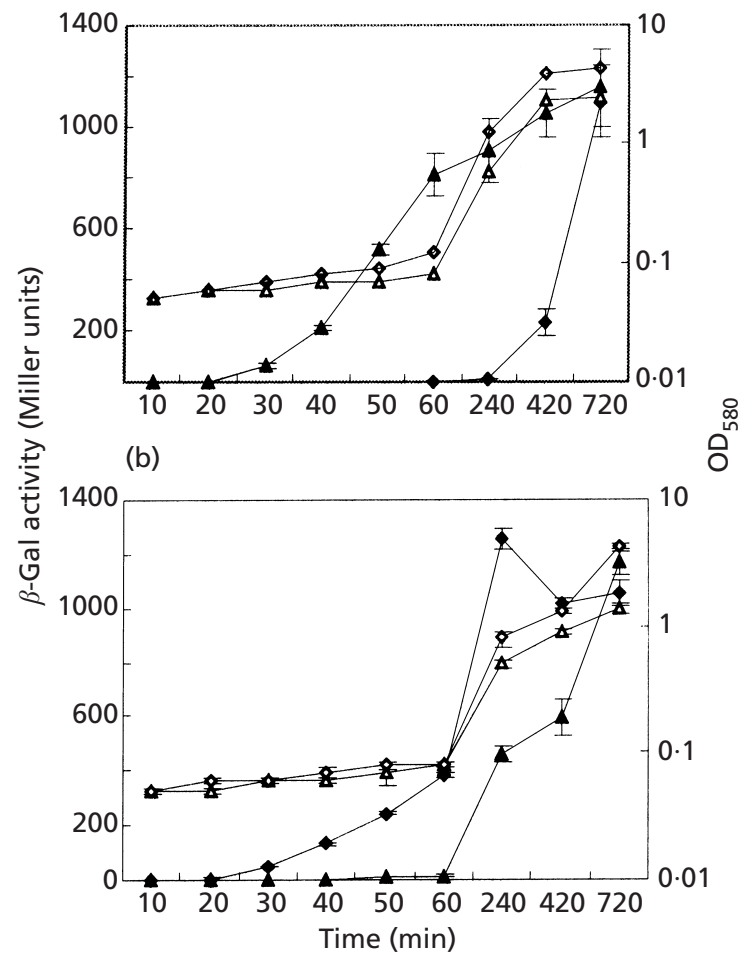

Fig. 4. Effect of growth medium composition on transcription from the pheBA promoter (a) and from the catBCA promoter (b). Bacteria were grown in MOPS minimal medium containing glucose. Sodium benzoate was added as a source of inducer. $\beta$-Gal activity (filled symbols) was measured in exponentially growing cells of $P$. putida strain PaW85 grown either in the presence $(\Delta, \diamond)$ or absence $(\boldsymbol{\Delta}, \triangle)$ of amino acids $(0.5 \%$ CAA) in MOPS minimal medium. The growth curves of $P$. putida cells are indicated by open symbols. The standard deviation is shown in the figure.

presence of CAA in MOPS minimal medium, the level of transcription from the pheBA promoter was very low during the first $4 \mathrm{~h}$ of cultivation (Fig. $4 \mathrm{a}$ ). The repression effect of CAA on transcription activation was also apparent on the catBCA promoter, but this promoter appeared to be less sensitive to the presence of CAA in the growth medium than the pheBA promoter. In the case of the catBCA promoter, expression of $\beta$-Gal activity was detectable $60 \mathrm{~min}$ after the addition of sodium benzoate (Fig. 4b).

Transcription from the $\sigma^{54}$-dependent Po promoter of the dimethylphenol $(d m p)$-degradation pathway is silenced in rich medium until amino acids become limiting (Sze \& Schingler, 1999). The requirement of (p)ppGpp for activation of transcription from the Po promoter was demonstrated using overexpression of RelA and E. coli (p)ppGpp-deficient mutants (Sze \& Schingler, 1999). We were not able to demonstrate the positive role of (p)ppGpp on transcriptional activation from the pheBA and catBCA promoters. The absence of an effect of (p)ppGpp on transcription from these promoters was confirmed by experiments where the
(p)ppGpp level was artificially increased either by adding $1 \mathrm{mM}$ serine hydroxamate into the growth medium or by using RelA overexpression plasmid pVI536 (Sze \& Schingler, 1999) in P. putida cells carrying the pheBAlac $Z$ transcriptional fusion in the chromosome (data not shown).

In the (p)ppGpp-dependent Po regulatory system, distinct groups of amino acids were not able to mediate the delay in transcription (Sze \& Schingler, 1999). We found that specific groups of amino acids could cause partial silencing of transcription from the pheBA and catBCA promoters. Bacteria were grown in MOPS minimal medium containing glucose for $60 \mathrm{~min}$ in the presence of sodium benzoate. No amino acids or a different number of amino acids (all 20,15 or 5) were added into the growth medium. The sets of amino acids were designed according to their biosynthetic pathways. The third group consisted of 5 amino acids (Asp, Asn, Glu, Gln and Ser) that are precursors for several other amino acids, and the second group contained the other 15 amino acids. In the absence of amino acids, the level of $\beta$-Gal activity was $950 \pm 44$ Miller units in the case of the pheBA promoter and $397 \pm 50$ Miller units in the case of the catBCA promoter. In the case of the pheBA promoter, no expression of $\beta$-Gal activity could be detected when all amino acids were added, but the presence of 5 and 15 amino acids allowed partial expression of this promoter: the $\beta$-Gal activities were $91 \pm 3$ and $196 \pm 20$ Miller units, respectively. The repressive effect of amino acids on transcription from the cat $B C A$ promoter was lower than that observed with the pheBA promoter. The $\beta$-Gal activity was $12 \pm 2 \cdot 5$ Miller units when all amino acids were added into the growth medium and it was approximately half of that observed without amino acids: $178 \pm 18$ and $199 \pm 50$ Miller units in the presence of 5 and 15 amino acids, respectively. This also indicates that the physiological control on the pheBA and catBCA promoters mediated by the presence of amino acids might be different from the mechanism related to stringent response. The occurrence of the partial silencing effect by different groups of amino acids on the transcription from the pheBA and catBCA promoters excludes the possibility that one particular amino acid could mediate this effect.

At this stage the mechanism by which the presence of amino acids causes repression of these two promoters is unclear. Data presented in Fig. 4 and results obtained in experiments with different sets of amino acids show that the exponential silencing of the pheBA promoter is stronger than that of the cat $B C A$ promoter. The nucleotide sequences of the pheBA and catBCA promoters are similar, but not identical. Differences become most apparent on sequences located downstream from the CatR-binding sites and -35 hexamers of the promoters (Fig. 1). Analysis of the locations of regulatory sites of $\sigma^{70}$-dependent promoters has revealed that repressors usually bind downstream from -30 (Gralla et al., 1996). It is possible that a hypothetical repressor protein could bind to the pheBA and catBCA promoters with different efficiency due to sequence 
differences of the target DNA. However, despite the minor differences in the expression level of the pheBA and $c a t B C A$ promoters under certain growth conditions, the general mechanisms for physiological control of these promoters seem to be well conserved to coordinate the expression of the hybrid plasmid-chromosomeencoded pathway for phenol degradation in P. putida.

\section{ACKNOWLEDGEMENTS}

We thank V. Shingler for kindly providing plasmid pVI536. We also thank T. Alamäe and R. Hõrak for critically reading the manuscript. This work was supported by grants 2323 and 4481 from the Estonian Science Foundation.

\section{REFERENCES}

Bayley, S. A., Duggleby, C. J., Worsey, M. J., Williams, P. A., Hardy, K. G. \& Broda, P. (1977). Two modes of loss of the TOL function from Pseudomonas putida mt-2. Mol Gen Genet 154, 203-204.

Bradford, M. M. (1976). A rapid and sensitive method for the quantitation of microgram quantities of protein utilizing the principle of protein-dye binding. Anal Biochem 72, 248-254.

Carmona, M., Rodriguez, M. J., Martinez-Costa, O. \& de Lorenzo, V. (2000). In vivo and in vitro effects of (p)ppGpp on the $\sigma^{54}$ promoter $\mathrm{Pu}$ of the TOL plasmid of Pseudomonas putida. J Bacteriol 182, 4711-4718.

Carter, P., Bedouelle, H. \& Winter, G. (1985). Improved oligonucleotide site-directed mutagenesis using M13 vectors. Nucleic Acids Res 13, 4431-4443.

Cases, I. \& de Lorenzo, V. (1998). Expression systems and physiological control of promoter activity in bacteria. Curr Opin Microbiol 1, 303-310.

Cases, I. \& de Lorenzo, V. (2000). Genetic evidence of distinct physiological regulation mechanisms in the $\sigma^{54} \mathrm{Pu}$ promoter of Pseudomonas putida. J Bacteriol 182, 956-960.

Cases, I., de Lorenzo, V. \& Perez-Martin, J. (1996). Involvement of $\sigma^{54}$ in exponential silencing of the Pseudomonas putida TOL plasmid Pu promoter. Mol Microbiol 19, 7-17.

Cases, I., Perez-Martin, J. \& de Lorenzo, V. (1999). The IIA ${ }^{\mathrm{Ntr}}$ (PtsN) protein of Pseudomonas putida mediates the C source inhibition of the $\sigma^{54}$-dependent Pu promoter of the TOL plasmid. J Biol Chem 274, 15562-15568.

Chugani, S. A., Parsek, M. R., Hershberger, C. D., Murakami, K., Ishihama, A. \& Chakrabarty, A. M. (1997). Activation of the catBCA promoter: probing the interaction of CatR and RNA polymerase through in vitro transcription. J Bacteriol 179, 2221-2227.

Gralla, J. D. \& Collado-Vides, J. (1996). Organization and function of transcription regulatory elements. In Escherichia coli and Salmonella typhimurium: Cellular and Molecular Biology, 2nd edn, pp. 1232-1245. Edited by F. C. Neidhardt, R. Curtiss, III, J. L. Ingraham, E. C. C. Lin, K. B. Low, B. Magasanik, W. S. Reznikoff, M. Riley, M. Schaechter \& H. E. Umbarger. Washington, DC: American Society for Microbiology.

Hanahan, D. (1983). Studies on transformation of Escherichia coli with plasmids. J Mol Biol 166, 557-580.

Harayama, S. \& Timmis, K. N. (1989). Catabolism of aromatic hydrocarbons by Pseudomonas. In Genetics of Bacterial Diversity, pp. 151-174. Edited by D. A. Hopwood \& K. E. Chater. London: Academic Press.
Harwood, C. S. \& Parales, R. E. (1996). The $\beta$-ketoadipate pathway and the biology of self-identity. Annu Rev Microbiol 50, 553-590.

Hengge-Aronis, R. (1999). Interplay of global regulators and cell physiology in the general stress response of Escherichia coli. Curr Opin Microbiol 2, 148-152.

Hengge-Aronis, R. (2000). The general stress response in Escherichia coli. In Bacterial Stress Responses, pp. 161-178. Edited by G. Storz \& R. Hengge-Aronis. Washington, DC: American Society for Microbiology.

Herrero, M., de Lorenzo, V. \& Timmis, K. N. (1990). Transposon vectors containing non-antibiotic resistance selection markers for cloning and stable chromosomal insertion of foreign genes in gram-negative bacteria. J Bacteriol 172, 6557-6567.

Hõrak, R. \& Kivisaar, M. (1998). Expression of the transposase gene $\operatorname{tn} p A$ of $\operatorname{Tn} 4652$ is positively affected by integration host factor. J Bacteriol 180, 2822-2829.

Houghton, J. E., Brown, T. M., Appel, A. J., Hughes, E. J. \& Ornston, L. N. (1995). Discontinuities in the evolution of Pseudomonas putida cat genes. J Bacteriol 177, 401-412.

Kasak, L., Hõrak, R., Nurk, A., Talvik, K. \& Kivisaar, M. (1993). Regulation of the catechol 1,2-dioxygenase- and phenol monooxygenase-encoding pheBA operon in Pseudomonas putida PaW85. J Bacteriol 175, 8038-8042.

Kivisaar, M., Hõrak, R., Kasak, L., Heinaru, A. \& Habicht, J. (1990). Selection of independent plasmids determining phenol degradation in Pseudomonas putida and the cloning and expression of genes encoding phenol monooxygenase and catechol 1,2dioxygenase. Plasmid 24, 25-36.

Kivisaar, M., Kasak, L. \& Nurk, A. (1991). Sequence of the plasmidencoded catechol 1,2-dioxygenase-expressing gene, pheB, of phenol-degrading Pseudomonas sp. strain EST1001. Gene 98, $15-20$.

de Lorenzo, V., Herrero, M., Jakubzik, U. \& Timmis, K. N. (1990). Mini-Tn5 transposon derivatives for insertion mutagenesis, promoter probing, and chromosomal insertion of cloned DNA in gram-negative eubacteria. J Bacteriol 172, 6568-6572.

de Lorenzo, V., Cases, I., Herrero, M. \& Timmis, K. N. (1993). Early and late responses of TOL promoters to pathway inducers: identification of postexponential promoters in Pseudomonas putida with lacZ-tet bicistronic reporters. J Bacteriol 175, 6902-6907.

Marques, S., Holtel, A., Timmis, K. N. \& Ramos, J. (1994). Transcriptional induction kinetics from the promoters of the catabolic pathways of TOL plasmid pWW0 of Pseudomonas putida for metabolism of aromatics. J Bacteriol 176, 2517-2524.

Marques, S., Manzanera, M., Gonzalez-Perez, M.-M., Gallegos, M.-T. \& Ramos, J. L. (1999). The XylS-dependent Pm promoter is transcribed in vivo by RNA polymerase with $\sigma^{32}$ or $\sigma^{38}$ depending on the growth phase. Mol Microbiol 31, 1105-1113.

Miller, J. H. (1992). A Short Course in Bacterial Genetics. Cold Spring Harbor, NY: Cold Spring Harbor Laboratory.

Miller, V. L. \& Mekalanos, J. J. (1988). A novel suicide vector and its use in construction of insertion mutations: osmoregulation of outer membrane proteins and virulence determinants in Vibrio cholerae requires toxR. J Bacteriol 170, 2575-2583.

Neidhardt, F. C., Bloch, P. L. \& Smith, D. F. (1974). Culture medium for enterobacteria. J Bacteriol 119, 736-747.

Ojangu, E., Tover, A. \& Kivisaar, M. (2000). Effects of combination of different -10 hexamers and downstream sequences on stationary phase-specific sigma factor $\sigma^{\mathrm{S}}$-dependent transcription in Pseudomonas putida. J Bacteriol 182, 6707-6713.

Parsek, M. R., Kivisaar, M. \& Chakrabarty, A. M. (1995). 
Differential DNA bending introduced by the Pseudomonas putida LysR-type regulator, CatR, at the plasmid-borne $p h e B A$ and chromosomal catBC promoters. Mol Microbiol 15, 819-828.

Ramos, J. L., Marques, S. \& Timmis, K. N. (1997). Transcriptional control of the Pseudomonas TOL plasmid catabolic operons is achieved through an interplay of host factors and plasmidencoded regulators. Annu Rev Microbiol 51, 341-373.

Rothmel, R. K., Aldrich, T. L., Houghton, J. E., Coco, W. M., Ornston, L. N. \& Chakrabarty, A. M. (1990). Nucleotide sequencing of Pseudomonas putida catR: positive regulator of the catBC operon is a member of LysR family. J Bacteriol 172, 922-931.

Rothmel, R. K., Shinabarger, D. L., Parsek, M. R., Aldrich, T. L. \& Chakrabarty, A. M. (1991). Functional analysis of the Pseudomonas putida regulatory protein CatR: transcriptional studies and determination of the CatR DNA-binding site by hydroxylradical footprinting. J Bacteriol 173, 4717-4724.

Sharma, R. C. \& Schimke, R. T. (1996). Preparation of electrocompetent E. coli using salt-free growth medium. BioTechniques 20, 42-44.
Sze, C. C. \& Shingler, V. (1999). The alarmone (p)ppGpp mediates physiological-responsive control at the $\sigma^{54}$-dependent Po promoter. Mol Microbiol 31, 1217-1228.

Sze, C. C., Moore, T. \& Shingler, V. (1996). Growth phasedependent transcription of the $\sigma^{54}$-dependent Po promoter controlling the Pseudomonas-derived (methyl)phenol $d m p$ operon of pVI150. J Bacteriol 178, 3727-3735.

Tover, A., Zernant, J., Chugani, S. A., Chakrabarty, A. M. \& Kivisaar, M. (2000). Critical nucleotides in the interaction of CatR with the pheBA promoter: conservation of the CatR-mediated regulation mechanisms between the pheBA and catBCA operons. Microbiology 146, 173-183.

Yuste, L., Canosa, I. \& Rojo, F. (1998). Carbon-source-dependent expression of the PalkB promoter from the Pseudomonas oleovorans alkane degradation pathway. J Bacteriol 180, $5218-5226$.

Received 11 December 2000; revised 19 March 2001; accepted 12 April 2001. 